\title{
El liderazgo transformacional en el trabajo docente: Colegio Mier y Pesado, un estudio de caso
}

\section{Transformational Leadership in Teaching: Mier y Pesado School, a Case Study}

\author{
Mónica del Carmen Meza-Mejía \\ Escuela de Pedagogía, \\ Universidad Panamericana \\ Ciudad de México, D.F., México \\ mmeza@up.edu.mx \\ Irma María Flores-Alanís ${ }^{2}$ \\ Facultad de Filosofía y Letras \\ Universidad Autónoma de Nuevo León \\ Monterrey, México \\ irma.floresal@uanl.edu.mx
}

Recibido: 7 noviembre 2012 Aceptado: 13 marzo $2014 \quad$ Corregido: 25 marzo 2014

\begin{abstract}
Resumen: Este trabajo de investigación parte de la premisa que en toda institución educativa, las personas docentes son líderes y como tales, han de gestionar la dinámica que se da en el salón de clases y hacer que las cosas sucedan. Es un estudio de caso que se apega a una metodología exploratoria-descriptiva sobre los rasgos de liderazgo transformacional (LT) que perciben tener los docentes del Colegio Mier y Pesado, ubicado en la ciudad de México. Las investigadoras plantean como pregunta de investigación: ¿qué rasgos de LT perciben tener la población docente?, y deriva en dos hipótesis: 1) a mayor nivel educativo del docente, mayor es el número de LT que percibe tener; 2) a mayor antigüedad en la institución, mayor número de rasgos de LT perciben tener los docentes. El instrumento de recolección de datos utilizado fue el cuestionario. Entre los resultados se encontró que no hay evidencia de que exista una relación significativa entre los rasgos del liderazgo transformacional del grupo docente con su antigüedad laboral o el grado máximo de estudios. Entre las limitaciones puede señalarse que al utilizarse una muestra pequeña, las correlaciones no fueron significativas para las hipótesis planteadas. Sin embargo, puede vislumbrarse la aplicación del cuestionario con una población más grande.
\end{abstract}

\section{Palabras clave:}

Liderazgo, Liderazgo Transformacional, trabajo docente, grado de estudios.

Abstract: The present research is founded on the premise that in a school, the teacher is a leader and must be perceived as such to manage the classroom in order to make things happen. The case under

\footnotetext{
$1 \quad$ Doctora en Educación por Universidad de Navarra, España; Maestría en Alta Dirección de Empresas por el Instituto Panamericano de Alta Dirección de Empresas; Maestría en Pedagogía por la Universidad Panamericana; Licenciada en Pedagogía por la Universidad Panamericana. Actualmente es Profesora de tiempo completo la Escuela de Pedagogía de la Universidad Panamericana, México. Pedagoga con trayectoria académica como docente e investigadora, principalmente en temas de educación y trabajo, desde la teoría de la educación.

2 Doctora en Educación (Ed.D.) por Loyola University Chicago; Maestría en Formación y Capacitación de Recursos Humanos (M.A.) por la Universidad Autónoma de Nuevo León; Licenciada en Pedagogía por la Universidad Autónoma de Nuevo León. Actualmente es Profesora Titular de la Facultad de Filosofía y Letras de la Universidad Autónoma de Nuevo León en México, así como Profesora Invitada en la Universidad Panamericana Campus Ciudad de México, en México. Pedagoga con trayectoria académica como docente e investigadora, principalmente en temas de educación y trabajo docente a nivel medio superior y superior, en procesos de enseñanza y aprendizaje.
} 
study uses an exploratory-descriptive methodology to analyze whether the teachers Mier y Pesado School, located in Mexico City, perceive having any of the characteristics from Transformational Leadership (TL). Regarding this question, the researchers' perception leads to two hypotheses: 1) the higher the teachers' educational level is, the more TL characteristics they have; 2) the more teaching experience teachers have, the more LT characteristics they have acquired throughout the years. The data collection instrument used was a questionnaire. Among the limitations of the study, it can be noted that the correlations -between teaching experience, educational level and LT characteristics-were not significant for the proposed hypotheses due to the small sample size used. However, future research is encouraged by applying the questionnaire with a larger sample.

Keywords: Leadership, Transformational Leadership, teaching, educational level.

\section{Marco de referencia}

\section{a. Marco teórico-conceptual}

La tendencia en la formación docente tiene como eje central desarrollar las competencias necesarias para contribuir a la tarea de elevar la calidad del aprendizaje de sus alumnos. De ahí, que en una institución educativa, el liderazgo docente es clave. Dentro del desarrollo de todo sistema, el liderazgo es una de las cuestiones más importantes. En este marco referencial, el trabajo docente se presenta como una tarea compleja en tanto "desafía el profesionalismo de los profesores, motivándolos a ejercer un liderazgo que les permita guiar, motivar y movilizar a los demás participantes del proceso de enseñanza-aprendizaje, para promover efectivos y significativos aprendizajes en sus alumnos para que alcancen su pleno desarrollo personal, en sana y justa convivencia con los demás" (González y González, 2008, p. 37). Así, el trabajo docente cuya misión principal radica en promover el acto educativo, requiere del desarrollo de un liderazgo eficaz para alcanzar su cometido.

Parafraseando a Ginebra (1995, pp. 5-6), el liderazgo es la capacidad de influir en las personas para que se esfuercen voluntaria y entusiastamente en la consecución de las metas; asimismo, es la capacidad de guiar y estimular hacia el logro de resultados. Así entendido, el liderazgo en las organizaciones educativas y vinculado a la docencia, resulta fundamental en: 1) la interpretación y uso que se hace de los aspectos institucionales y en este sentido, los docentes son actores de conexión clave dentro de la organización para alcanzar metas; 2) la gestión de los planes formativos en el conjunto de la institución dando coherencia y continuidad a la intervención de las diversas instancias, en donde los docentes se vislumbran claramente como agentes de innovación y de cambio, toda vez que sus planes de acción educativa suponen la adecuación a los tiempos y variables del entorno, así como la intervención para coadyuvar en el desarrollo de las personas educandas (Zabalza, 2002, pp. 93-94). De tal manera, se puede afirmar con Murillo Torrecilla (2006, p. 11) que si se quiere cambiar las escuelas, y con ello, mejorar la educación, se necesita contar con personas que ejerzan un liderazgo desde su interior, que inicien, impulsen, faciliten gestiones y coordinen el proceso de transformación, que posean una preparación técnica adecuada y, sobre todo, con una actitud y un compromiso con la escuela, la educación y la sociedad capaces de ponerse al frente del proceso de cambio. 
Por lo anteriormente expuesto, las investigaciones sobre el liderazgo en las instituciones educativas se han multiplicado en estos últimos años, quizá como un indicador no sólo del interés y la relevancia de la temática, sino también por la preocupación que se tiene de tener evidencias para la mejora escolar, aunque en sus inicios, entre de las décadas de los sesenta y los setenta, la investigación sobre el liderazgo educativo, se desarrolló como una adaptación a la educación de la investigación sobre liderazgo en general, como afirma Yukl (2006). Un hito fundamental en la investigación sobre el liderazgo educativo es el que se orienta a configurar modelos de estilos de liderazgo, para contribuir a mejorar la educación. De ahí surgieron los conceptos de liderazgo: «transformacional» y «transaccional», en el marco de las teorías centradas en la relación entre el líder y el colaborador (teorías relacionales del liderazgo). Un exponente de estas teorías, Burns (1978) define el liderazgo como el proceso por el cual los líderes inducen y animan a los seguidores a conseguir ciertos objetivos que encarnan los valores, motivaciones, necesidades y expectativas de ambas partes, líder y seguidores. La relación entre ambos sujetos se sustenta en la reciprocidad mutua de las verdaderas necesidades que han de ser atendidas (citado por Cardona y García-Lomardía, 2009, p. 54). Así, Burns (1978) refiere que los estilos de liderazgo transaccional y transformacional, son extremos opuestos de un continuo. Sin embargo, Bass (1985), quien también se ubica en las teorías relacionales del liderazgo, considera ambos estilos como dimensiones separadas, lo que permite al líder ser transaccional/transformador, ambas cosas a la vez o ninguna de ellas. Siguiendo las ideas de Bass (1985), en ambos casos, se está ante una relación de influencia entre el líder y el colaborador, pues los dos intentan cambios reales que reflejan intereses mutuos.

Este estudio retoma el paradigma liderazgo transaccional/transformacional porque se considera que ambas proyecciones de liderazgo, resultan consustanciales para que la escuela esté en condiciones de cumplir su misión formativa, como sugiere Zabalza (2002, p.96). El liderazgo transformacional es un proceso orientador, que motiva a los seguidores en la dirección de los objetivos marcados, clarificando los requisitos. La autoridad de este paradigma de liderazgo se construye a partir de recompensas.

Por ello, el personal docente ejerce un liderazgo transaccional con el alumnado en la medida en la que orienta su liderazgo en hacer concesiones a los intereses personales de los educandos mediante refuerzos positivos o negativos. En contraste, el liderazgo transformacional, da sentido a lo que se hace e inspira a las personas a trascender sus propios intereses por un bien mayor. Este tipo de liderazgo se construye a partir de la confianza con la finalidad de mejorar los niveles de desempeño y satisfacción de las personas, estimulando el aprendizaje y al mismo tiempo, un mayor grado de compromiso, participación y lealtad de los individuos y de los grupos. De ahí que el personal docente ejerza un liderazgo transformacional cuando dirige su influencia a potenciar en el alumnado el interés hacia logro, la apertura continua al aprendizaje y les anima a trascender su interés individual con miras a un bien común. En la actividad docente el LT es más significativo ya que dirige al alumnado hacia la excelencia educativa. Un binomio docente-alumno motivado, se torna en una poderosa herramienta de innovación y de cambio capaz de transformar las realidades más adversas en oportunidades de desarrollo (Prieto y Zambrano, 2005, p. 8). Si bien el liderazgo es un continuo, es evidente el impacto y la importancia de ejercer un liderazgo docente más transformacional que transaccional. 
El líder transformacional potencia los deseos de los seguidores, que logran metas y un desarrollo continuo, al mismo tiempo que promueve la mejora de los grupos y la organización En lugar de ceder a las demandas individuales de sus seguidores, promueve una mayor altura de miras en cada persona y pone énfasis en los asuntos clave para la organización (Vázquez Alatorre, 2013, p. 78). Al mismo tiempo incrementa la confianza de los seguidores y gradualmente los mueve hacia el crecimiento y desarrollo de sí mismos (Bass y Riggio, 2006).

El LT, conforme a la teoría de Bass, contiene algunos factores. Pascual, Villa y Auzmendi (1993, p. 24), los resumen de la siguiente manera:

- Carisma. Capacidad de entusiasmar, transmitir confianza, generar respeto, sentido de finalidad y orgullo por la tarea realizada.

- Consideración individual. Capacidad para atender las necesidades individuales de las personas y dar un trato diferenciado a cada una de ellas; así como la capacidad para orientar y apoyar el logro de metas de cada persona, haciéndolas agentes de su propia formación.

- Estimulación intelectual. Capacidad para encontrar nuevos enfoques a viejos problemas; es decir, creatividad e innovación para resolver problemas.

- Inspiración. Capacidad para identificar las expectativas en la gente, generar visión de un futuro posible, despertar el optimismo y el entusiasmo para alcanzar las metas.

Además, para efectos de esta investigación, se añadieron tres factores, que se consideraron relevantes porque se refieren al ámbito educativo, mismos que se tomaron del esquema de Bernal (2000, p. 445):

- Tolerancia psicológica. Capacidad para resolver conflictos.

- Participación. Capacidad para trabajar en equipo.

- Actuación del/de la docente. Capacidad para asumir riesgos.

Si bien en la acción se encuentran interrelacionados estos siete factores, desde un punto de vista teórico se pueden desglosar. El propio Bass (1999), apunta en sus trabajos empíricos, que los factores están presentes de manera conjunta en un todo identificado como LT.

En este tipo de estudios, es clásico ya el cuestionario diseñado por Bass y Avolio (1995), el Multifactor Leadership Questionnaire MLQ, por sus siglas, que identifica la unidad de observación de una investigación con los factores del liderazgo transformacional. El MLQ es una escala tipo Likert, compuesta por ítems que miden variables llamadas de primer orden. Una de ellas es la de LT. Dentro de esta variable se encuentran otras, llamadas de segundo orden, que se identifican con los factores de la teoría del LT. 


\section{b. Marco contextual}

El estudio se realizó en en el Colegio Mier y Pesado, una institución de 75 años de antigüedad, ubicada en la Delegación Gustavo A. Madero, de la Ciudad de México. Es una escuela particular, confesional y de educación diferenciada (sólo mujeres). La escuela cuenta con 1500 alumnas divididas en las secciones de Preescolar, Primaria, Secundaria y Preparatoria, distribuidos como se muestra en la Tabla 1. La dirección general está a cargo de una religiosa, que estudió la liceniciatura en Pedagogía y la maestría en Educación. Asimismo, hay una directora por sección y un director técnico por sección. Un subdirector de Secundaria. En Primaria además, hay dos coordinadoras. En cada sección, menos en Preescolar, hay una prefecta por nivel. En total 100 profesores configuran el plantel docente. La escuela se apoya con 18 personas en lo administrativo y 26 personas intendentes.

Tabla 1

División de grupos por sección

\begin{tabular}{|c|c|c|c|}
\hline Sección & Grado & $\begin{array}{l}\text { No. de } \\
\text { grupos }\end{array}$ & $\begin{array}{c}\text { Total de } \\
\text { grupos }\end{array}$ \\
\hline \multirow[t]{3}{*}{ Preescolar } & $1^{\circ}$ & 4 & \\
\hline & $2^{\circ}$ & 3 & 11 \\
\hline & $3^{\circ}$ & 3 & \\
\hline \multirow[t]{6}{*}{ Primaria } & $1^{\circ}$ & 4 & \\
\hline & $2^{\circ}$ & 4 & \\
\hline & $3^{\circ}$ & 3 & \\
\hline & $4^{\circ}$ & 4 & 23 \\
\hline & $5^{\circ}$ & 4 & \\
\hline & $6^{\circ}$ & 4 & \\
\hline \multirow[t]{3}{*}{ Secundaria } & $1^{o}$ & 4 & \\
\hline & $2^{\circ}$ & 4 & 11 \\
\hline & $3^{\circ}$ & 3 & \\
\hline \multirow[t]{3}{*}{ Preparatoria } & $1^{o}$ & 2 & \\
\hline & $2^{\circ}$ & 2 & 6 \\
\hline & $3^{\mathrm{o}}$ & 2 & \\
\hline
\end{tabular}

Nota: Elaborado por las autoras.

\section{Metodología}

El estudio de caso es un trabajo cualitativo que sigue el paradigma $\mathrm{N}=1$, que significa analizar la particularidad y la complejidad de un caso singular, y donde dicho análisis 
permita llegar a comprender el caso en circunstancias complejas. Esta metodología, se auxilia de diversos instrumentos de recolección de información, como entrevistas y cuestionarios, entre otros. Este es un trabajo de tipo descriptivo-explicativo, para dar respuesta a una pregunta de investigación: ¿Qué rasgos de LT perciben tener los docentes? La pregunta de investigación conllevó a dos hipótesis:

1.- A mayor nivel educativo del docente, mayor es el número de LT, que percibe tener.

2.- A mayor antigüedad en la institución, mayor número de rasgos de LT perciben tener los docentes.

Como herramienta para la recolección de la información se utilizó un cuestionario tipo Likert (anexo 1), que se retomó del esquema del MLQ (por sus siglas en inglés, Cuestionario de Liderazgo Multifactorial), adaptado por Bernal (2000, pp. 441-458) para el análisis del LT de los profesores de instituciones educativas. El cuestionario original consta de 124 preguntas. El cuestionario aplicado se modificó a partir de una selección aleatoria de preguntas ponderadas, según los factores de liderazgo, quedando finalmente un instrumento de 32 preguntas (ver anexo). Cabe señalar que se realizó un estudio piloto donde participaron 20 profesores de una institución similar.

Para la investigación Se solicitó la participación de todos los profesores del CMyP (unidad de observación) que impartieron clase en el año académico inmediato anterior, lo que significó una población de 98 profesores. El grupo de profesores fue dividido en tres categorías de acuerdo con su estatus laboral: TC (48.0\%), MT (10.2\%) y PH (38.8\%) (Tabla 2).

\section{Tabla 2.}

Categoría laboral del docente

\begin{tabular}{cc}
\hline Categoría docente & Porcentaje \\
\hline Tiempo completo & $\mathbf{4 8 . 0}$ \\
Medio tiempo & 10.2 \\
Por horas & 38.8 \\
No contestó & 3.0 \\
Total & $100.0 \%$ \\
\hline
\end{tabular}

Nota: Elaborado por las autoras.

\section{Resultados}

En la tabla 3, se observa que el grado máximo de estudios de los docentes es el de licenciatura, lo que no se consideró es si el docente cuenta con el título de licenciado: En segundo lugar, están aquellos que tienen tanto el grado de licenciado como el de normal básica. 
Tabla 3.

Grado máximo de estudios de los profesores

\begin{tabular}{cc}
\hline Grado máximo de estudios & Porcentaje \\
\hline Licenciatura & $\mathbf{4 4 . 9}$ \\
Normal Básica & 17.3 \\
Normal y licenciatura & 23.5 \\
Especialidad y/o maestría & 10.2 \\
Doctorado & 2.0 \\
No contestó & 2.0 \\
Total & $\mathbf{1 0 0 . 0 \%}$ \\
\hline
\end{tabular}

Nota: Elaborado por las autoras.

Los profesores del CMyP tienen una antigüedad promedio de más de 13 años, reflejando que están a la mitad de su etapa laboral, pues el total de años mínimos laborados como docentes debe ser de 30 años de servicio, antes de su jubilación. Sobre la antigüedad, Mendoza, Ortiz y Rosell (2006, pp. 30-31), señalan en su estudio que, el LT se afecta por el grado académico y por la experiencia profesional del profesor, porque la profesionalización de la actividad docente incrementa las conductas transformacionales.

En la tabla 4 se observa que al igual que en muchas instituciones donde se trabaja la educación como profesión, el número de docentes femeninos es mayor que el de docentes masculinos, y el CMyP no es la excepción, sobre todo, en relación a ser una escuela diferenciada sólo de mujeres. Si bien en este estudio no se indagó sobre la predominancia de los rasgos del LT en cuanto a las diferencias de género, estudios como los de Mendoza, Ortiz y Rosell ya citados, afirman que las mujeres tienden a ser más transformacionales en su liderazgo que los hombres (2006, p. 28).

Tabla 4.

Número de profesores en relación al sexo

\begin{tabular}{cc}
\hline Sexo & Porcentaje \\
\hline Mujeres & $\mathbf{7 7 . 6}$ \\
Hombres & 26.7 \\
No contesto & 1.0 \\
Total & $\mathbf{1 0 0 . 0} \%$ \\
\hline
\end{tabular}

Nota: Elaborado por las autoras.

Ahora bien, los datos obtenidos sobre los factores de LT, se resumen en la tabla 6 y se comenta cada uno por separado, a continuación. 
Del factor carisma, el $56.4 \%$ afirma que frecuentemente busca entusiasmar, dar confianza e inspirar respeto a sus estudiantes. El cuestionario, abarcaba ocho preguntas de este factor. Las otras preguntas arrojaron los siguientes resultados: más del $50 \%$ asegura tener confianza en sí mismos. El $31.8 \%$ expresa que a menudo se siente cercano a sus estudiantes. Sobre los ítems que se refieren a aspectos de coherencia, honestidad y optimismo, los profesores perciben frecuentemente la presencia de esos rasgos en su trabajo docente; también resaltan su capacidad de escucha al señalar que frecuentemente están abiertos a atender a sus estudiantes. Lo que el factor carisma aglutina en la teoría del LT, resume una actitud pedagógica básica que parte del nivel de consciencia que tiene el docente sobre sus competencias personales y profesionales. Así, el profesor se percibe capaz de demostrar su tacto pedagógico, su conocimiento profundo del ambiente de aprendizaje que generan sus acciones y la inteligencia emocional que subyace en la interacción educativa.

Sobre la consideración individual. La tabla 5 señala que el $43.0 \%$ a menudo forma y aconseja a sus estudiantes; y el $42.8 \%$ percibe que lo hace frecuentemente. Al sumar los datos de las opciones "a menudo" y "frecuentemente", se establece que más del $80 \%$ de los profesores perciben que este rasgo está presente en su actuar docente. El profesor que desarrolla este factor transformacional, no concibe al alumno genérico (aunque evidentemente detecta aspectos comunes entre sus estudiantes). Se adapta al contexto, a la historia de cada sujeto, comprende sus motivaciones y necesidades de aprendizaje.

La tabla 5, reporta sobre la estimulación intelectual, que el $44.7 \%$ de los profesores frecuentemente se perciben utilizando nuevos enfoques para ir solucionando los problemas que se les presentan; pueden estar centrados en estrategias de enseñanza y en actividades innovadoras que fomenten el aprendizaje colaborativo y el desarrollo de competencias. Actualmente los profesores afrontan retos que les implica innovar en el proceso de enseñanza-aprendizaje, pues las tendencias y recomendaciones que marcan los organismos internacionales para el aprendizaje del siglo XXI, requieren de nuevos enfoques y de apertura a la innovación y la mejora continua en la enseñanza.

La inspiración como factor de LT reporta un porcentaje de $89.5 \%$ en el nivel más alto de la escala del cuestionario, al sumar el $46.0 \%$ de las respuestas dadas como "frecuentemente" y el 43.5\% de "a menudo". Por tanto, la capacidad para aumentar el optimismo y el entusiasmo de los estudiantes para lograr una mayor implicación de ellos en el aula, parece ser vital para los docentes del CMyP.

Los datos de la tabla 5 muestran que el $50.3 \%$ de los profesores frecuentemente recurren a la tolerancia psicológica para lograr un ambiente áulico agradable y así resolver los conflictos que en ella se generan. Saber convivir, es una competencia a tener presente en un entorno en donde diferentes conductas antisociales, como el bullying, están presentes.

Acerca de la participación docente el $45.5 \%$ contestó "frecuentemente" y el 42.3\%, "a menudo". En este sentido, los profesores se perciben construyendo un liderazgo compartido entre los estudiantes y ellos mismos. De lo anterior, se puede inferir que el trabajo en equipo está presente en la actividad docente del CMyP. Ahora bien, este factor también arrojó información en el mismo sentido positivo, pues para este estudio, el 100\% de profesores contestaron el cuestionario, de este dato, se infiere que los profesores son altamente participativos. 
Por último, está la actuación del/de la docente, que enfatiza el sentido de cambio del proceso educativo, donde juntos el profesor y el estudiante se comunican, corren riesgos y buscan la eficacia. En la tabla 6 se visualiza que tomando en cuenta las respuestas expuestas en "frecuentemente" y "a menudo", el 88.0\% es la respuesta docente en cuanto a este factor de LT.

Tabla 5.

Rasgos de liderazgo transformacional

\begin{tabular}{|c|c|c|c|c|c|c|c|}
\hline Factores en $\%$ & 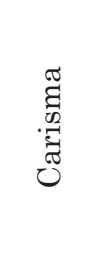 & 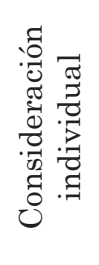 & 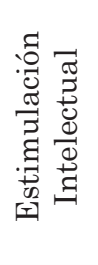 & 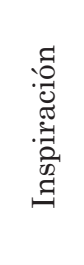 & 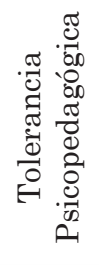 & 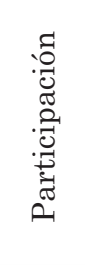 & 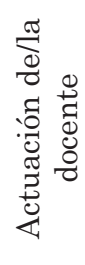 \\
\hline \multicolumn{8}{|l|}{ Escala } \\
\hline No contestó & 0.5 & 0.3 & 0.7 & 0.5 & 0.0 & 0.0 & 0.8 \\
\hline Frecuente- & 56.4 & 42.8 & 44.7 & 46.0 & 50.3 & 45.5 & \\
\hline mente & & & & & & & 47.7 \\
\hline A menudo & 31.8 & 43.0 & 41.7 & 43.5 & 41.0 & 42.3 & 40.3 \\
\hline A veces & 7.9 & 11.5 & 10.3 & 7.5 & 6.0 & 9.5 & 8.8 \\
\hline $\begin{array}{l}\text { Raras } \\
\text { veces }\end{array}$ & 1.4 & 0.0 & 0.3 & 0.5 & 0.7 & 0.8 & 0.3 \\
\hline Nunca & 0.0 & 0.0 & 0.3 & 0.0 & 0.0 & 0.0 & 0.0 \\
\hline Total & 100 & 100 & 100 & 100 & 100 & 100 & 100 \\
\hline
\end{tabular}

Nota: Elaborado por las autoras.

Sobre la pregunta de investigación 1, la acentuación de los rasgos de LT de los profesores del CMyP, se muestra en la tabla 6. De ella, podría inferirse que existe cierta interrelación entre todos los factores que conforman el perfil del LT, como apuntan los estudios empíricos de Bass, destacando que la tolerancia psicológica es el rasgo de liderazgo transformador, que más predomina en estos docentes. Ello indica que en el trabajo docente, el entorno de aprendizaje, es especialmente relevante para los profesores del CMyP. 
Tabla 6.

Rasgos acentuados de LT en los profesores

\begin{tabular}{cc}
\hline Rasgo de liderazgo transformacional & Porcentaje \\
\hline Carisma & 90.0 \\
Consideración individual & 88.0 \\
Estimulación intelectual & 89.9 \\
Inspiración & 91.0 \\
Tolerancia psicológica & $\mathbf{9 3 . 0}$ \\
Participación & 89.0 \\
Actuación del / de la docente & 90.0 \\
\hline
\end{tabular}

Nota: Elaborado por las autoras.

De acuerdo con el análisis de correlación (Tabla 7), se observa que los rasgos de liderazgo transformacional no son significativamente diferentes de 0 , por lo tanto, no hay una correlación entre las variables de rasgos de LT y el grado máximo de estudios de los profesores del CMyP. Se rechaza la hipótesis de investigación.

Tabla 7.

Análisis de correlación de Pearson

\begin{tabular}{llc}
\hline & Grado máximo de estudios & \\
\hline Carisma & Correlación de Pearson & -.041 \\
& Sig. (bilateral) & .692 \\
& N & 98 \\
Consideración Individual & Correlación de Pearson & .013 \\
& Sig. (bilateral) & .901 \\
& N & 98 \\
Estimulación Intelectual & Correlación de Pearson & .079 \\
& Sig. (bilateral) & .442 \\
Inspiración & N & 98 \\
& Correlación de Pearson & .063 \\
Tolerancia psicológica & Sig. (bilateral) & .535 \\
& N & 98 \\
& Correlación de Pearson & -.030 \\
Participación & Sig. (bilateral) & .772 \\
& N & 98 \\
& Correlación de Pearson & .071 \\
Actuación de/la docente & Cig. (bilateral) & .485 \\
& Correlación de Pearson & 98 \\
& Sig. (bilateral) & .045 \\
& N & .660 \\
& & 98 \\
\hline
\end{tabular}

Nota: Elaborado por las autoras. 
Al trabajar la segunda hipótesis que señala: "a mayor antigüedad en la institución, mayor número de rasgos de LT perciben tener los docentes", tampoco se observó correlación, interpretando que no se corresponden la antigüedad del personal docente con la percepción de la posesión de rasgos de LT por los mismos, como se evidencia en la Tabla 8.

\section{Tabla 8.}

Correlación de los rasgos de LT de los profesores y la antigëdad docente

\begin{tabular}{|c|c|c|c|c|c|c|c|c|}
\hline & 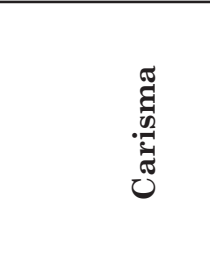 & 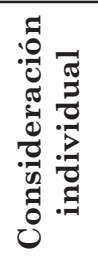 & 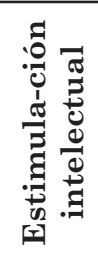 & 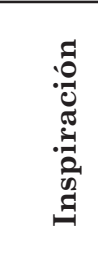 & 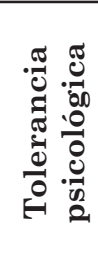 & 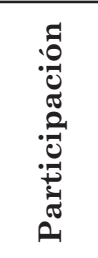 & 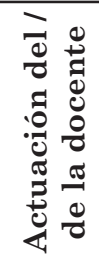 & \\
\hline \multirow[t]{2}{*}{ Antigüedad } & $\begin{array}{l}\text { Correlación } \\
\text { de Pearson }\end{array}$ & 0.137 & -0.032 & 0.037 & 0.046 & 0.024 & 0.091 & 0.169 \\
\hline & Significancia & 0.181 & 0.751 & 0.719 & 0.656 & 0.812 & 0.091 & 0.096 \\
\hline
\end{tabular}

Nota: Elaborado por las autoras.

Para finalizar el análisis de los resultados, se señala que el personal docente CMyP sí se percibe como líder en su salón de clases; Bass y Riggio (2006) establecen que el liderazgo implica desempeño y afán de logro; los profesores buscan como líderes, desempeñarse competentemente y cumplir con los objetivos educativos. También, ejercer su liderazgo al ponderar una interacción abierta y respetuosa entre los actores del proceso enseñanzaaprendizaje, como lo asientan González y González (2008, p. 37). Finalmente, los profesores como líderes, afrontan los retos que se le presentan en las tareas de la profesión y el entorno a partir de la reflexión sobre su quehacer cotidiano. Se considera entonces una interrelación entre los factores de LT, como los apunta la teoría de Bass sobre el liderazgo transformacional del profesor.

\section{Conclusiones y limitaciones}

El liderazgo transformacional como lo afirman Cardona y Wilkinson (2010, p. 45), apunta a que el profesor es un agente de cambio, que influye en las personas (estudiantes) para que se esfuercen voluntaria y entusiastamente en la consecución de las metas y/o tareas establecidas. La teoría de Bass, sustenta que el líder (en este caso, el docente) es aquel que potencia el interés hacia el logro y la formación continua de sus colaboradores (sus estudiantes), les anima a trascender en su interés individual con miras a un bien común: grupal, organizacional y/o social. 
El LT en la teoría de Bass (1985), aglutina factores, que se han retomado en este estudio, más los que Bernal (2000) suma: carisma, consideración individual, estimulación intelectual, inspiración, tolerancia psicológica, participación y actuación docente. Estos factores si bien pueden estudiarse de manera individual, están interrelacionados en la práctica, lo que varía en cada docente, es la intensidad en la que se presenta cada rasgo.

El docente va desarrollando el liderazgo transformacional con cierto sello institucional, que se da al establecer el plan de mejora del centro educativo; es decir, cada curso de capacitación, va encaminado a desarrollar de manera implícita en el docente factores del liderazgo transformacional. A su vez, se observa en términos generales, que las variables personales de los profesores (edad, sexo, formación profesional, experiencia docente, y antigüedad) no inciden en la percepción de liderazgo ejercida como profesor.

Al hablar de las limitaciones de la investigación, se señala que al dirigirse el estudio a una población pequeña y en una sola institución educativa, no permite que las correlaciones sean de mayor significancia, y esto conlleva a considerarse como un estudio parcial.

$\mathrm{Al}$ ser un estudio parcial, se recomienda extender a otros actores del $\mathrm{CMyP}$, como al personal directivo, al administrativo, y a la comunidad estudiantil, para conocer la manera en que perciben el liderazgo transformacional del docente del CMyP, esto sería bajo la premisa de hacer cruces de información y obtener nuevas vertientes de investigación.

\section{Anexo}

\section{Cuestionario: Factores de LT aplicado al personal docente del CMyP}

\begin{tabular}{|c|c|c|c|c|c|c|}
\hline \multicolumn{2}{|r|}{ La escala que utiliza el cuestionario es: } & $\begin{array}{c}\text { Nunca } \\
0\end{array}$ & $\begin{array}{c}\text { Rara } \\
\text { vez } \\
1\end{array}$ & $\begin{array}{c}\text { A } \\
\text { veces } \\
2\end{array}$ & $\begin{array}{c}\underset{\text { menudo }}{3} \\
3\end{array}$ & $\begin{array}{c}\text { Frecuentemente } \\
4\end{array}$ \\
\hline 1 & $\begin{array}{l}\text { Procuro que la comunicación entre mis estudiantes como la } \\
\text { comunicación entre mis colegas funcione adecuadamente. }\end{array}$ & & & & & \\
\hline 2 & Soy consciente de las consecuencias que mis acciones provocan. & & & & & \\
\hline 3 & $\begin{array}{l}\text { Proporciono nuevos enfoques ante situaciones que son para mí } \\
\text { problemáticas. }\end{array}$ & & & & & \\
\hline 4 & Me siento a gusto de trabajar con mis grupos. & & & & & \\
\hline 5 & Soy coherente con lo que digo y hago. & & & & & \\
\hline 6 & Me anima reflexionar sobre cómo puedo mejorar mi trabajo docente. & & & & & \\
\hline 7 & Suelo admitir si he cometido algún error o he hecho algo mal. & & & & & \\
\hline 8 & Soy optimista. & & & & & \\
\hline 9 & $\begin{array}{l}\text { Suelo apoyar a mis estudiantes adecuadamente en los problemas que } \\
\text { les crea algún tipo de incertidumbre. }\end{array}$ & & & & & \\
\hline 10 & $\begin{array}{l}\text { Me gusta participar en todo tipo de procesos que se genera en la } \\
\text { institución (rediseños, evaluaciones institucionales, entre otras). }\end{array}$ & & & & & \\
\hline 11 & $\begin{array}{l}\text { Me mantengo al tanto de los intereses, prioridades y necesidades de } \\
\text { mis estudiantes. }\end{array}$ & & & & & \\
\hline 12 & $\begin{array}{l}\text { Puedo ser riguroso (a) y estricto (a), pero siempre agradable en el } \\
\text { trato. }\end{array}$ & & & & & \\
\hline 13 & Soy capaz de escuchar. & & & & & \\
\hline
\end{tabular}




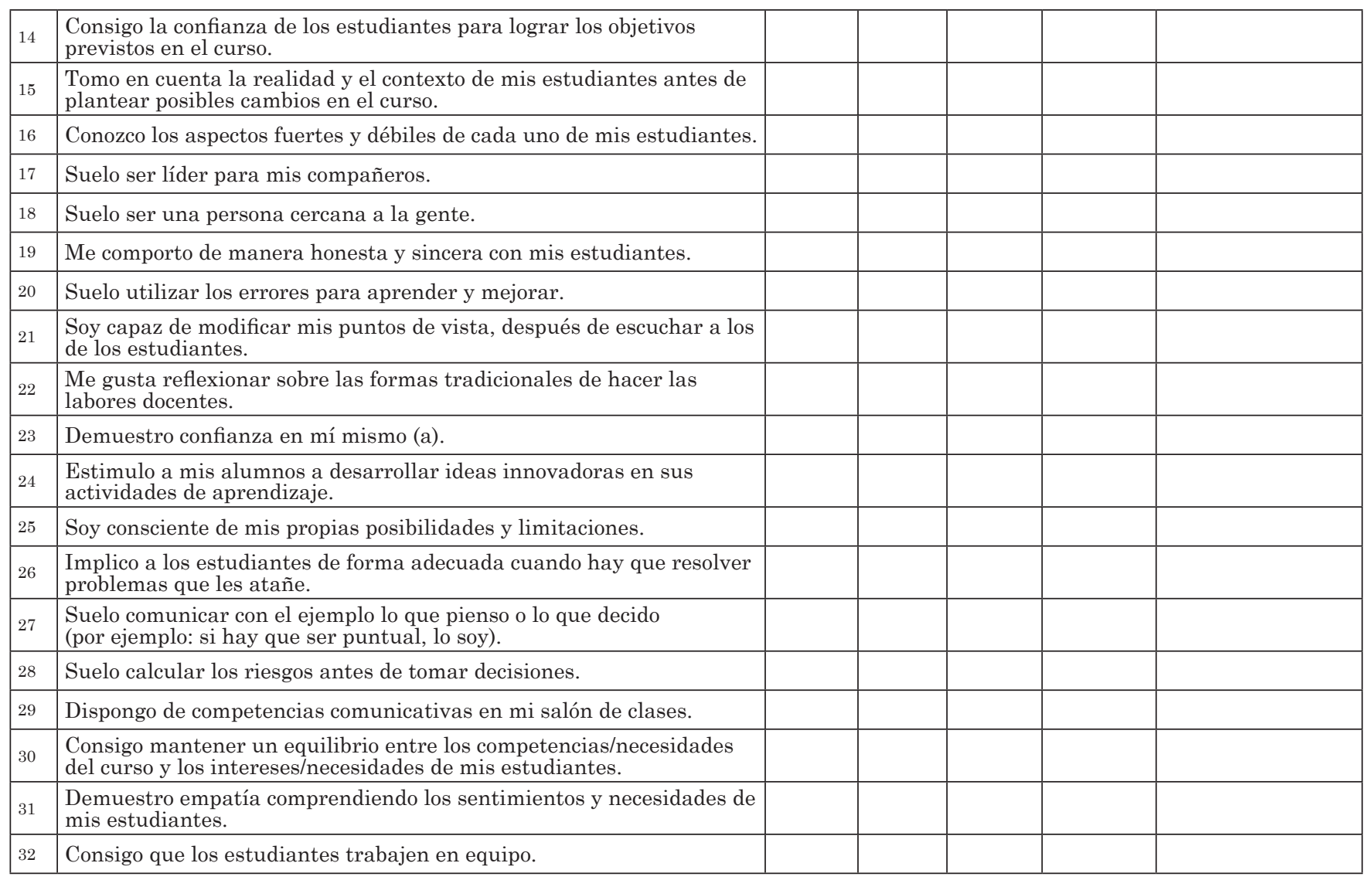

\section{Distribución de las preguntas del cuestionario conforme al factor de LT}

\section{Factor de liderazgo transformacional}

\section{Preguntas de cuestionario}

$4,5,8,12,13,17,18,19,23$ у 27

$11,16,22$ y 31

3,6 y 24

10 y 14

9,20 y 21

$1,26,30$ y 32

$2,7,15,25,28$ у 29
Total de preguntas

Consideración individual.

Estimulación intelectual.

Inspiración.

Tolerancia psicológica.

Participación.

Actuación del / de la docente.

4

3

2

3

4

6

32

TOTAL PREGUNTAS DEL CUESTIONARIO 


\section{Referencias}

Bass, B. M. (1985). Leadership and Performance Beyond Expectations [Liderazgo y actuación más allá de las expectativas]. New York: Free Press.

Bass, B. M. (1999). Current developments in transformational leadership [Desarrollos actuales en liderazgo transformacional] en The Psychologist-Manager Journal, 3(1), 5-21.-Philadelphia: Psychology Press.

Bass, B. M. y Avolio, B. J. (1995). The Multifactor Leadership Questionnaire (MLQ) [E] Multifactor Leadership Questionnaire (MLQ)]; Redwood City: Mind Garden. Recuperado de www.mindgarden.com

Bass, B. M. y Riggio, R. (2006). Transformational leadership [Liderazgo transformacional]. New Jersey: Lawrence Erlbaum Associates Publishers.

Bernal, J. L. (2000). Cuestionario sobre liderazgo transformacional. Aportaciones desde una invetigación. En Liderazgo y organizaciones que aprenden. Congreso Internacional sobre Dirección de Centros Educativos, Bilbao: Universidad de Deusto, pp. 441-458.

Burns, J. (1978). Leadership. [Liderazgo]. New York: Harper \& Row.

Cardona, P. y García-Lombardía, P. (2009). Cómo desarrollar las competencias de liderazgo (4ta ed.). Pamplona: Eunsa.

Cardona, P. y Wilkinson, H. (2010). Creciento como líder (2da ed.). Pamplona: Eunsa.

Ginebra, J. (1995). El Liderazgo y la acción. Mitos y realidades.México: McGraw-Hill Interamericana.

González, O. y González, O. (noviembre 2007-febrero 2008). Percepciones de los estudiantes universitarios, frente al liderazgo docente en Revista Mexicana de Orientación educativa, 5(13), 37-43.

Mendoza, I. A., Ortiz Arévalo, M. F. y Rosell, P. (enero-julio 2007). Dos décadas de investigación y desarrollo en liderazgo transformacional en Revista del Centro de Investigación. Universidad La Salle, 7(27), 25-41.

Murillo Torrecilla, F. J., (2006). Una dirección escolar para el cambio: del liderazgo transformacional al liderazgo distribuido, en Revista Electrónica Iberoamericana sobre Calidad, Eficacia y Cambio en educación, 4(4e), 11-24. Recuperado de http://www. rinace.net/arts/vol4num4e/art2_htm.htm 
Pascual, R., Villa, A. y Auzmendi, E. (1993). El liderazgo transformacional en los centros docentes. Bilbao, Mensajero.

Prieto, A. y Zambrano, E. (enero-abril 2005). Ética y liderazgo transformacional en la docencia, en Telos, 7(1), 81-91. Universidad Rafael Belloso Chacín, Maracaibo, Venezuela.

Sheard, A. G. y Kakabadse, A. P. (2004) A process perspective on leadership and team development [Perspectiva del proceso de desarrollo de liderazgo y equipo], en Journal of Management Development, 23 (1), 7-10.

Vázquez Alatorre, A. (2013). Interdependencia entre el liderazgo transformacional, cultura organizacional y cambio educativo: una reflexión en Revista Iberoamericana sobre Calidad, Eficacia y Cambio en Educación, 11(1), 73-91.

Yukl, G. A. (2006). Leadership in organizations [Liderazgo en organizaciones], Upper Saddle River, New Jersey, Prentice Hall.

Zabalza, M. A. (2002). La enseñanza universitaria. El escenario y sus protagonistas. Madrid: Narcea. 